\title{
Rectal, central venous, gastric and bladder pressures versus esophageal pressure for the measurement of cough strength: a prospective clinical comparison
}

\author{
Lluís G. Aguilera', Lluís Gallart ${ }^{1 *}$ D, Juan C. Álvarez ${ }^{1}$, Jordi Vallès ${ }^{1}$ and Joaquim Gea ${ }^{2}$
}

\begin{abstract}
Background: Cough pressure, an expression of expiratory muscle strength, is usually measured with esophageal or gastric balloons, but these invasive catheters can be uncomfortable for the patient or their placement impractical. Because pressure in the thorax and abdomen are expected to be similar during a cough, we hypothesized that measurement at other thoracic or abdominal locations might also be similar as well as useful in clinical scenarios. This study aimed to compare cough pressures measured at thoracic and abdominal sites that could serve as alternatives to esophageal pressures $\left(P_{E S}\right)$.
\end{abstract}

Methods: Nine patients scheduled for laparotomy were asked to cough as forcefully as possible from total lung capacity in supine position. Three cough maneuvers were performed while $P_{E S}$ (the gold standard) as well as gastric, central venous, bladder and rectal pressures $\left(\mathrm{P}_{\mathrm{GA}}, \mathrm{P}_{\mathrm{CV}}, \mathrm{P}_{\mathrm{BL}}\right.$, and $\mathrm{P}_{\mathrm{REC},}$, respectively) were measured simultaneously. The intraclass correlation coefficient (ICC) was used to evaluate the repeatability of the measurements in each patient at each site and evaluate agreement between alternative sites $\left(P_{G A}, P_{C V}, P_{B L}\right.$ and $\left.P_{R E C}\right)$ and $P_{E S}$. Bland-Altman plots were used to compare $P_{\text {es }}$ and the measurements at the other sites.

Results: Median (first quartile, third quartile) maximum pressures were as follows: $P_{E S} 112(89,148), P_{G A} 105(92,156)$, $P_{\mathrm{CV}} 102(91,149), \mathrm{P}_{\mathrm{BL}} 118(93,157)$, and $\mathrm{P}_{\mathrm{REC}} 103(88,150) \mathrm{cmH}_{2} \mathrm{O}$. The ICCs showed excellent within-site repeatability of the measurements $(p<0.001)$ and excellent agreement between alternative sites and $P_{E S}(p<0.004)$. The Bland-Altman plots showed minimal differences between $P_{E S \prime} P_{G A}, P_{C V}$ and $P_{R E C}$. However, $P_{B L}$ was higher than the other pressures in most patients, and the difference between $\mathrm{P}_{\mathrm{ES}}$ and $\mathrm{P}_{\mathrm{BL}}$ was slightly larger.

Conclusions: Cough pressure can be measured in the esophagus, stomach, superior vena cava or rectum, since their values are similar. It can also be measured in the bladder, although the value will be slightly higher. These results potentially facilitate the assessment of dynamic expiratory muscle strength with fewer invasive catheter placements in most hospitalized patients, thus providing an option that will be particularly useful in those undergoing thoracic or abdominal surgery.

Trial registration: NCT02957045 registered at November 7, 2016. Retrospectively registered.

Keywords: Respiratory muscles [A02.633.567.900], Abdominal muscles [A02.633.567.050], Cough [C23.888.852.293], Laparotomy [E04.406]

\footnotetext{
* Correspondence: LGallart@parcdesalutmar.cat

${ }^{1}$ Department of Anesthesiology, Parc de Salut MAR, Institut Hospital del Mar d'Investigacions Mèdiques (IMIM), Universitat Autònoma de Barcelona (UAB), Passeig Maritim 25, 08003 Barcelona, Spain

Full list of author information is available at the end of the article
}

(c) The Author(s). 2018 Open Access This article is distributed under the terms of the Creative Commons Attribution 4.0 International License (http://creativecommons.org/licenses/by/4.0/), which permits unrestricted use, distribution, and reproduction in any medium, provided you give appropriate credit to the original author(s) and the source, provide a link to the Creative Commons license, and indicate if changes were made. The Creative Commons Public Domain Dedication waiver (http://creativecommons.org/publicdomain/zero/1.0/) applies to the data made available in this article, unless otherwise stated. 


\section{Background}

Cough is a physiological response whose purpose is to eliminate secretions from the airway tract. Thus, the inability to cough forcefully enough to be effective would increase the risk of pulmonary complications such as atelectasis or pneumonia. This inability is observed in neuromuscular or respiratory diseases and is particularly likely after abdominal or thoracic surgery, when pain, surgical injury and/or the residual effect of anesthetics come into play [1-3].

Because a cough maneuver can be made voluntarily and easily by a patient without training, cough pressure measurements at various sites can be obtained readily in clinical situations, in contrast to the often used maximum mouth expiratory pressure recorded during a static artificial maneuver $[4,5]$, which must be learned. Cough pressure is usually measured with balloon catheters that record maximum gastric or esophageal pressures $\left(\mathrm{P}_{\mathrm{GA}}, \mathrm{P}_{\mathrm{ES}}\right)$ [4-6]. It is not always practical to use these catheters outside research scenarios, however, and they can cause discomfort $[4,7]$. Furthermore, they cannot be used in certain situations, such as during postoperative recovery from gastrointestinal surgery. Other points of measurement that might potentially be used to reflect cough pressure include central venous pressure $\left(\mathrm{P}_{\mathrm{CV}}\right)$, which has been used as an alternative to $\mathrm{P}_{\mathrm{ES}}[8]$; bladder pressure $\left(\mathrm{P}_{\mathrm{BL}}\right)$, which has been compared to $\mathrm{P}_{\mathrm{GA}}$ $[8,9]$; and rectal pressure $\left(\mathrm{P}_{\mathrm{REC}}\right)$, which has been used to measure intra-abdominal pressure as an alternative to $\mathrm{P}_{\mathrm{BL}}$ [10]. However, none of these pressures have yet been used to evaluate expiratory muscle strength, even though many hospitalized patients have a catheter already placed in the superior vena cava or in the bladder.

We hypothesized that these catheters could be suitable for measuring cough pressure without compromising patient comfort, as occurs with the placement of a gastric or esophageal balloon. We also reasoned that placement of a rectal balloon catheter, which does not cause the nausea associated with esophageal or gastric balloons, could also measure cough pressure comfortably. If these hypotheses are correct, wider clinical use of cough pressure to reflect respiratory muscle strength and possible risk of respiratory compromise might be facilitated. The aim of this study was to evaluate the use of $\mathrm{P}_{\mathrm{CV}}, \mathrm{P}_{\mathrm{BL}}$, and $\mathrm{P}_{\mathrm{REC}}$ as alternatives to $\mathrm{P}_{\mathrm{ES}}$ or $\mathrm{P}_{\mathrm{GA}}$ for the measurement of cough pressure.

\section{Methods}

\section{Patients}

Adult patients scheduled for open-midline laparotomy for colon cancer surgery, which required placement of central venous and bladder catheters, were enrolled prospectively. Exclusion criteria included rectal surgery, chronic obstructive pulmonary disease [11], neuromuscular disorders [1], chronic pain, and factors that could impede an adequate recording of the research protocol variables.
The study was approved by the clinical research ethics committee of Parc de Salut Mar (CEIC-Parc de Salut Mar) and by the Spanish Agency for Medicines and Health Products (AEMPS). All patients signed an informed consent form before entering the study, and we provided each with an insurance policy to cover care in the event of adverse events related to the procedures.

\section{Interventions and measurements}

All patients underwent forced spirometry measurement (Datospir 500, SIBEL, Barcelona, Spain) the day before surgery. Reference values were those for a Mediterranean population [11].

Pressures were measured with catheters placed in the esophagus, stomach, superior vena cava, bladder and rectum as follows:

- $\mathrm{P}_{\mathrm{ES}}$ and $\mathrm{P}_{\mathrm{GA}}$ were measured with compliance balloon catheters (esophageal catheter Jaeger 720,199, Viasys Healthcare, Hoechberg, Germany) as previously described [5]. The catheters were introduced nasally under local anesthesia and the balloons were filled with 1-2 $\mathrm{mL}$ of air.

- $\mathrm{P}_{\mathrm{CV}}$ was measured from the distal port of a doublelumen catheter (CV-26702-E, Arrow, Erding, Germany) placed through the subclavian or internal jugular veins [12]. Correct positioning was checked with the $\mathrm{P}_{\mathrm{CV}}$ waveform [13].

- $\mathrm{P}_{\mathrm{BL}}$ was measured with a transurethral (Foley) catheter inserted after the bladder was drained and $50 \mathrm{~mL}$ of a $0.9 \%$ saline solution was instilled [8, 14, 15].

- $\mathrm{P}_{\mathrm{REC}}$ was measured with a compliance balloon inserted $10 \mathrm{~cm}$ inside the rectum and filled with $5 \mathrm{~mL}$ of air [10].

All the pressure curves were displayed on a screen and recorded with a data acquisition system (Acknowledge and MP100, Biopac, Santa Barbara, CA, USA) for off-line analysis. Patients lay in supine position and all the pressure transducers were calibrated and aligned with the axillary midline.

The correct placement of all catheters was assessed by asking the patient to perform a sharp sniff and a cough maneuver while the researcher monitored the signal on the computer screen [5]. Once all catheters were inserted and after a 3-min resting period, baseline respiratory pattern and pressures were recorded.

Cough pressure was then measured at all points. Patients were asked, always by the same researcher (L.G.A.), to cough as forcefully as possible [5] from total lung capacity (TLC). Two or three trials were performed to allow the patient to practice the maneuver before data recording started. Next, the pressures generated by three valid maneuvers, separated by pauses of 5-10 s, were recorded and 
the difference between the baseline pressure at relaxed end-expiratory lung volume and the peak pressure attained during each cough from TLC was registered, as previously described [4-6]. Maneuvers were considered valid if the patient followed the instruction to cough and the expected cough pressure waveform was observed on the screen [5].

Soon after the end of the protocol, the patients were asked to indicate which catheters caused the least and the most discomfort.

\section{Statistical analysis}

The sample size was calculated to allow us to detect an intraclass correlation coefficient (ICC) value defined as excellent [16] with a statistical power of $80 \%$ based on the range of cough pressure values obtained at the different sites during an earlier study [6]. The ICC was used as the measure of the reliability within each patient of the cough pressure measurements at each site and to reflect agreement between alternative sites and $\mathrm{P}_{\mathrm{ES}}$. Bland-Altman plots of mean differences were used to compare $\mathrm{P}_{\mathrm{GA}}, \mathrm{P}_{\mathrm{CD}}, \mathrm{P}_{\mathrm{BL}}$ and $\mathrm{P}_{\mathrm{REC}}$ with the gold-standard $\left(\mathrm{P}_{\mathrm{ES}}\right)$ [17]. $\mathrm{P}_{\mathrm{ES}}$ rather than $\mathrm{P}_{\mathrm{GA}}$ was chosen as the gold standard because the former is measured in the chest, where a cough effort becomes effective. Maneuvers that generated the maximum $\mathrm{P}_{\mathrm{ES}}$ were chosen for comparison between sites. That maximum value was then compared to the pressures generated by the patient during the same maneuver at each of the other sites.
Table 1 Demographic, anthropometric and functional data

\begin{tabular}{|c|c|}
\hline Age (yrs) & $66(53,72)$ \\
\hline Gender (male/female) & $6 / 3$ \\
\hline ASA class (I/II/III) & $0 / 9 / 0$ \\
\hline Height (cm) & $167(157,169)$ \\
\hline Weight (kg) & $66(59,72)$ \\
\hline $\mathrm{FEV}_{1}(\mathrm{~L} / \mathrm{min})$ & $2.36(2.2,3.48)$ \\
\hline $\mathrm{FEV}_{1}$ (\% pred) & $91(84,99)$ \\
\hline $\mathrm{FVC}(\mathrm{L})$ & $2.81(2.73,4.17)$ \\
\hline FVC (\% pred) & $94(82,97)$ \\
\hline $\mathrm{FEV}_{1} / \mathrm{FVC}(\%)$ & $81(78,106)$ \\
\hline
\end{tabular}

Data are presented as median (first quartile,third quartile) or number of subjects ASA American Society of Anesthesiologists physical status classification system, $F E V$ forced expiratory volume in one second, FVC forced vital capacity, \% pred percentage of the predicted value

A $p$ value of $<0.05$ was considered statistically significant in the ICC analysis. Statistical analysis was performed using IBM SPSS (IBM, Armonk, NY, USA) and STATA (STATA Corp., College Station, TX, USA) software.

\section{Results}

\section{Participants}

Eleven patients initially consented to participate in the study, but one withdrew consent as catheters were about to be inserted. Data from one patient were lost because
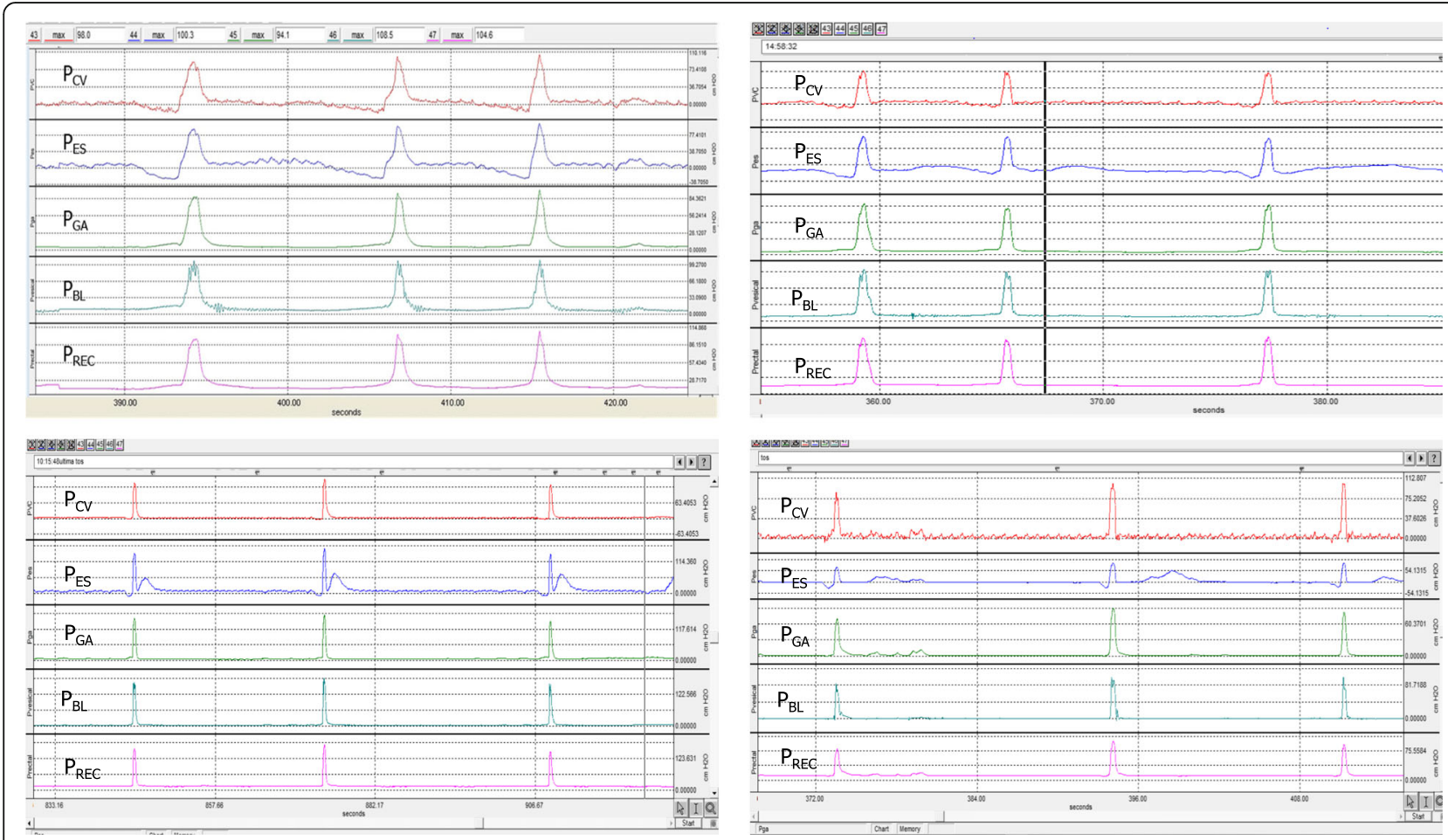

Fig. 1 Waveforms at all five measurement sites. Congruent waveforms recorded for four patients at all five measurement sites. $P_{C V}=c e n t r a l$ venous pressure; $\mathrm{P}_{\mathrm{ES}}=$ esophageal pressure; $\mathrm{P}_{\mathrm{GA}}=$ gastric pressure; $\mathrm{P}_{\mathrm{BL}}=$ bladder pressure; $\mathrm{P}_{\mathrm{REC}}=$ rectal pressure 
of technical problems. Thus, we analyzed data for nine patients. Their characteristics are summarized in Table 1. No adverse events were observed during the insertion of the catheters.

\section{Test results}

The cough pressure curves for all sites were congruent. Figure 1 shows the pressure curves for four patients.

The median (first quartile, third quartile) for the maximum $P_{E S}$ values for the nine patients was $112(89,148)$ $\mathrm{cmH}_{2} \mathrm{O}$. The other median cough pressures recorded at the same time as the maximum $\mathrm{P}_{\mathrm{ES}}$ values were as follows: $\mathrm{P}_{\mathrm{GA}} 105(92,156), \mathrm{P}_{\mathrm{cv}} 102(91,149), \mathrm{P}_{\mathrm{BL}} 118(93,157)$, and $\mathrm{P}_{\text {REC }} 103(88,150) \mathrm{cmH}_{2} \mathrm{O}$.

Individual measurements recorded for patients at each site are shown in Table 2. Bold-face values show the maneuvers that generated maximum $\mathrm{P}_{\mathrm{ES}}$.

The ICCs showed excellent repeatability between the three pressures recorded for each patient at each site (within-site repeatability) $(p<0.001)$ and excellent agreement between alternative sites and $\mathrm{P}_{\mathrm{ES}}(p<0.004)$ (Table 3$)$.

The median (first quartile, third quartile) differences between maximum $\mathrm{P}_{\mathrm{ES}}$ and pressures at other sites were as follows: $\mathrm{P}_{\mathrm{GA}} 0.7(-1.8,13.5), \mathrm{P}_{\mathrm{CV}}-2.1(-4.2,5.2), \mathrm{P}_{\mathrm{BL}}$ $5.8(4,12.9)$, and $\mathrm{P}_{\mathrm{REC}}-2.5(-9.5,12) \mathrm{cmH}_{2} \mathrm{O}$. Bland-Altman plots of the differences between $\mathrm{P}_{\mathrm{ES}}$ and each of the other pressures are shown in Fig. 2. $P_{C V}$ and $P_{R E C}$ were the alternative-site pressures that showed the best agreement (smallest average differences from $P_{E S}$ ). The average differences between $P_{B L}$ and $P_{E S}$ were slightly larger, $P_{B L}$ usually registering higher values than $P_{E S}$.

The rectal catheter was the least unpleasant for all the patients, whereas the ones introduced nasally into the esophagus and stomach were the most uncomfortable for seven patients ( 4 men and 3 women). The bladder catheter was the most uncomfortable for 2 men.

\section{Discussion}

This study demonstrates that cough pressure can be measured with central venous or rectal catheters as alternatives to conventional esophageal balloon catheters. Bladder catheters could also be used although recorded $\mathrm{P}_{\mathrm{BL}}$ values were systematically slightly higher than the gold-standard $\mathrm{P}_{\mathrm{ES}}$ values.

The ICC analysis indicated excellent repeatability between measurements at the same site, evidence of the precision of cough pressure measurements. In a few patients, cough pressures were slightly lower and differences among repeated maneuvers higher than for the other subjects, but in a series of continuous non-selected patients this minimal variability can be expected. In fact, $5-10 \%$ of adult outpatients cannot achieve reproducible pulmonary function results when repeated maneuvers are performed with coaching [18]. The largest source of within-subject
Table 2 Measurements of cough pressure at five sites

\begin{tabular}{|c|c|c|c|c|c|c|}
\hline $\begin{array}{l}\text { Patient } \\
\text { No. }\end{array}$ & $\begin{array}{l}\text { Maneuver } \\
\text { No. }\end{array}$ & $P_{E S}$ & $P_{G A}$ & $P_{c V}$ & $P_{B L}$ & $P_{\text {REC }}$ \\
\hline \multirow[t]{3}{*}{1} & 1 & 85.3 & 97.4 & 97.6 & 95.9 & 87 \\
\hline & 2 & 84.6 & 85.2 & 93.8 & 92 & 82.7 \\
\hline & 3 & 83.2 & 95 & 90.9 & 95.4 & 88.9 \\
\hline \multirow[t]{3}{*}{2} & 1 & 58.2 & 52.9 & 65.8 & 55.4 & 51.3 \\
\hline & 2 & 77.3 & 78 & 79.2 & 83.1 & 74.6 \\
\hline & 3 & 73.8 & 56.7 & 72.5 & 59.6 & 55.1 \\
\hline \multirow[t]{3}{*}{3} & 1 & 132.8 & 128.8 & 127.9 & 141.1 & 114.5 \\
\hline & 2 & 137.7 & 132.5 & 144.4 & 153.6 & 121.5 \\
\hline & 3 & 147.9 & 146.2 & 143.8 & 160.8 & 135.2 \\
\hline \multirow[t]{3}{*}{4} & 1 & 81.7 & 82.1 & 82.3 & 84.2 & 81.2 \\
\hline & 2 & 85.5 & 89.2 & 95 & 90.4 & 88.9 \\
\hline & 3 & 104 & 93.2 & 101.9 & 95.1 & 92.1 \\
\hline \multirow[t]{3}{*}{5} & 1 & 74.6 & 70 & 66.6 & 78.2 & 69.3 \\
\hline & 2 & 91.9 & 90.1 & 85.3 & 91 & 89.4 \\
\hline & 3 & 91.3 & 83.1 & 91.2 & 100.6 & 82.6 \\
\hline \multirow[t]{3}{*}{6} & 1 & 102.9 & 101.9 & 99.8 & 111.9 & 100.1 \\
\hline & 2 & 108.3 & 104.6 & 105.3 & 115.5 & 102.9 \\
\hline & 3 & 112.4 & 105.3 & 101.9 & 117.7 & 102.9 \\
\hline \multirow[t]{3}{*}{7} & 1 & 110.5 & 116.5 & 106.9 & 128.1 & 115.9 \\
\hline & 2 & 125.9 & 140.3 & 121.7 & 150.4 & 138.5 \\
\hline & 3 & 118.5 & 131.3 & 114.9 & 142 & 131 \\
\hline \multirow[t]{3}{*}{8} & 1 & 148.5 & 168.8 & 156.5 & 167.9 & 143.3 \\
\hline & 2 & 132.5 & 152.2 & 137.7 & 158 & 146.9 \\
\hline & 3 & 148.8 & 167.3 & 154 & 152.8 & 162.4 \\
\hline \multirow[t]{3}{*}{9} & 1 & 121.6 & 128.4 & 123.1 & 137.15 & 127.2 \\
\hline & 2 & 152.9 & 166.4 & 158.5 & 178.25 & 164.9 \\
\hline & 3 & 140.9 & 159.8 & 143.9 & 169.65 & 157.5 \\
\hline
\end{tabular}

Values are in $\mathrm{cmH}_{2} \mathrm{O}$. Bold-face values identify the maneuver that generated the maximum $\mathrm{P}_{\mathrm{ES}}$

$P_{E S}$ esophageal pressure, $P_{G A}$ gastric pressure, $P_{C V}$ central venous pressure, $P_{B L}$ bladder pressure, $P_{R E C}$ rectal pressure

variability of spirometry is improper performance of the test [19]. For these reasons, when voluntary maneuvers are recorded several measurements must always be performed and the best one will be chosen.

The ICC analysis also demonstrated excellent agreement between alternative sites and $\mathrm{P}_{\mathrm{ES}}$, and the BlandAltman plots showed small differences lacking clinical significance between $\mathrm{P}_{E S}$ and $\mathrm{P}_{\mathrm{GA}}, \mathrm{P}_{\mathrm{CV}}$ and $\mathrm{P}_{\mathrm{REC}}$. These data therefore suggest that the alternative measurements are reliably accurate. Thus, $\mathrm{P}_{\mathrm{CV}}$ or $\mathrm{P}_{\mathrm{REC}}$ would be valid candidates to choose as surrogates for $\mathrm{P}_{\mathrm{ES}}$. The greater difference between $P_{B L}$ and $P_{E S}$, on the other hand, shows that pressure behaves somewhat differently at the bladder, something the clinician would need to bear in mind. Furthermore, given that $P_{B L}$ was usually higher than $P_{E S}$, 
Table 3 Analysis of cough pressure measurement methods

\begin{tabular}{|c|c|c|c|}
\hline & ICC & $95 \% \mathrm{Cl}$ & $p$ value \\
\hline \multicolumn{4}{|c|}{ Within-site repeatability } \\
\hline$P_{E S}$ & 0.888 & $0.665-0.972$ & $p<0.001$ \\
\hline$P_{G A}$ & 0.905 & $0.730-0.976$ & $p<0.001$ \\
\hline$P_{c v}$ & 0.884 & $0.665-0.971$ & $p<0.001$ \\
\hline$P_{B L}$ & 0.906 & $0.718-0.976$ & $p<0.001$ \\
\hline$P_{\text {REC }}$ & 0.896 & $0.626-0.975$ & $p<0.001$ \\
\hline \multicolumn{4}{|c|}{ Between-site agreement } \\
\hline$P_{G A} V S P_{E S}$ & 0.943 & $0.784-0.987$ & $p<0.002$ \\
\hline$P_{C V} V S P_{E S}$ & 0.974 & $0.897-0.994$ & $p<0.001$ \\
\hline$P_{B L} V S P_{E S}$ & 0.913 & $0.687-0.979$ & $p<0.003$ \\
\hline$P_{\text {REC }}$ VS $P_{E S}$ & 0.951 & $0.811-0.988$ & $p<0.004$ \\
\hline
\end{tabular}

ICC intraclass correlation coefficient; $\mathrm{Cl}$ confidence interval

we can conclude that $\mathrm{P}_{\mathrm{BL}}$ was precise but less accurate than pressure measurements at the other alternative sites. This slight but systematic difference between $P_{E S}$ and $\mathrm{P}_{\mathrm{BL}}$ would mean that the bladder catheter would be the last-choice alternative to the esophageal catheter. $\mathrm{P}_{\mathrm{BL}}$ could nevertheless be useful in hospitalized patients who already have a bladder catheter in place, so as to avoid disturbing the patient by placing an additional one. The clinician must always take into account this fixed bias in relation to $P_{E S}$ when interpreting the pressures.
Although $\mathrm{P}_{\mathrm{GA}}$ has been used widely to reflect cough pressure in studies of respiratory muscle strength $[4-6,20]$ and was measured at the same time as $\mathrm{P}_{\mathrm{ES}}$ in this study, we designated $\mathrm{P}_{\mathrm{ES}}$ as the gold standard in the Bland-Altman analysis because it is recorded in the chest, where cough effort takes place. Cough pressures have fluctuated in previous studies of expiratory muscle strength measured with $\mathrm{P}_{\mathrm{ES}}$ or $\mathrm{P}_{\mathrm{GA}}[4,6,20-22]$ because the study populations varied. Higher cough pressures are observed in young, male, and tall subjects as well as in chronic coughers. We measured cough pressure in a specific surgical population, accounting for differences between our results and previously reported values.

Our study was performed under conditions relevant to clinical situations. Patients were in supine position, in which the pressure transducers were all at the same approximate level, favoring reliable comparison between measurement sites. Patients carrying central venous or Foley (bladder) catheters are usually confined to a bed. The cough maneuver was performed from TLC in order to achieve a standardized test measurement [4] and because it is usual to take a deep breath before a cough [5]. Maximum levels of respiratory muscle strength and hence pressure are expected from TLC $[5,23]$.

The practical implication of our study is that cough pressure can be measured using the technique that best fits the clinical condition of an individual patient. A central venous catheter would be the first choice if one has
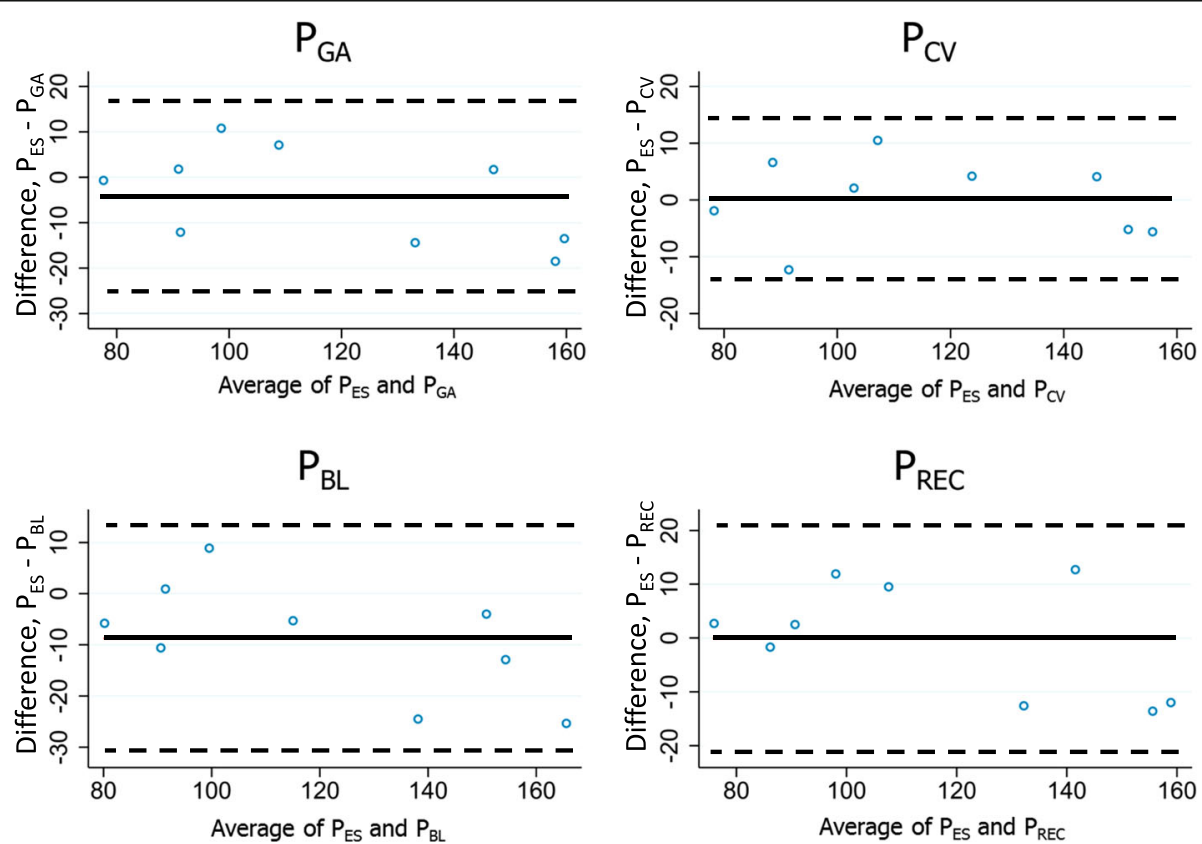

Fig. 2 Bland-Altman plots of differences between the maximum (optimal) $P_{E S}$ and the pressure measurements at the alternative sites. Circles indicate the difference between PES and the alternative measured pressures. The central line indicates the average difference between the compared measurements. The upper and lower (dotted) lines indicate the $95 \%$ confidence interval. Values are expressed in $\mathrm{cmH}_{2} \mathrm{O}$. $\mathrm{P}_{\mathrm{c} V}=c e n t r a l$ venous pressure; $\mathrm{P}_{E S}=$ esophageal pressure; $\mathrm{P}_{\mathrm{GA}}=$ gastric pressure; $\mathrm{P}_{\mathrm{BL}}=$ bladder pressure; $\mathrm{P}_{\mathrm{REC}}=$ rectal pressure 
been inserted. A bladder catheter could also be used provided the clinician bears in mind the larger difference between $\mathrm{P}_{\mathrm{BL}}$ and $\mathrm{P}_{\mathrm{ES}}$ discussed above. If no catheter has been inserted, a good choice would be a rectal catheter, which our patients found to be the least uncomfortable. $\mathrm{P}_{\mathrm{ES}}$ remains the gold standard, but its main disadvantage, that the insertion of a balloon catheter through the nose causes discomfort [4], was confirmed by our patients. In addition, discomfort can cause esophageal contractions that can impede correct measurement in a considerable percentage of patients [7]. For these reasons, esophageal and gastric balloons should probably be reserved for selected patients or volunteers under experimental conditions.

This study has limitations. The results probably cannot be extrapolated to patients with chronic cough, in whom voluntary cough pressure can be higher than in healthy individuals [20]. In addition, the results possibly cannot be extrapolated to scenarios in which cough is triggered by nerve stimulation [24] or to patient types we excluded. Similar results might well be obtained in these scenarios, but further studies would be needed to confirm that hypothesis.

Our results facilitate further investigation and patient management in many settings. An important scenario is the postoperative period after abdominal or thoracic surgery, where cough effort is reduced [1-3] and where patients are at risk of respiratory complications [25].

\section{Conclusions}

Pressure generated with a cough maneuver from TLC in supine position can be measured in the esophagus, stomach, superior vena cava, or rectum indistinctly. Bladder catheters could also be used, although the recorded pressures would usually be slightly higher than $\mathrm{P}_{\mathrm{ES}}$. These results support assessing expiratory muscle strength for clinical or research purposes without using an additional invasive catheter in most hospitalized patients. If no invasive catheter has already been placed for clinical purposes, a minimally invasive catheter can be chosen for assessing cough pressure.

\footnotetext{
Abbreviations

ASA: American Society of Anesthesiologists physical status classification system; Cl: Confidence interval; $\mathrm{FEV}_{1}$ : Forced expiratory volume in one second; FVC: Forced vital capacity; ICC: Intraclass Correlation Coefficient; $P_{B L}$ : Bladder pressure; $P_{C \mathrm{C}}$ : Central venous pressure; $P_{E S}$ : Esophageal pressure; $P_{G A}$ : Gastric pressure; $P_{R E C}:$ Rectal pressure; TLC: Total lung capacity
}

\section{Acknowledgments}

We thank the nurses of the surgical area of Hospital del Mar, Barcelona, for supporting our clinical management of patients. We also thank Xavier Duran and the staff of the Methodological and Biostatistical Advisory Service of the IMIM, Barcelona, for their cooperation. Mary Ellen Kerans gave advice on English language expression in a late version of the manuscript.

\section{Funding}

Supported by Spanish Health Ministry's research fund (Fondo de Investigaciones Sanitarias, reference FIS PI030127) and CIBERES, Instituto de Salud Carlos III (ISCIII), Spain.

\section{Availability of data and materials}

The datasets used and/or analyzed during the current study are available from the corresponding author on reasonable request.

\section{Authors' contributions}

LGA contributed to all parts of the design, analysis and reporting, and conducted the research protocol. LG proposed the initial aim of the study, analyzed data, and wrote the manuscript. JCA helped conduct the research protocol and collect data. JV supervised data collection and analysis. JG. advised and critically reviewed the manuscript. All authors approved the final manuscript and guarantee its integrity.

\section{Ethics approval and consent to participate}

This study was approved by our institutional review board (the clinical research ethics committee of Parc de Salut Mar-CEIC-Parc de Salut Mar), reference number 031554. It was also approved by the Spanish Agency for Medicines and Health Products (AEMPS), reference number 040073.

All patients signed an informed consent form before being enrolled in the study.

\section{Consent for publication}

Not applicable.

\section{Competing interests}

The authors declare that they have no competing interests.

\section{Publisher's Note}

Springer Nature remains neutral with regard to jurisdictional claims in published maps and institutional affiliations.

\section{Author details}

'Department of Anesthesiology, Parc de Salut MAR, Institut Hospital del Mar d'Investigacions Mèdiques (IMIM), Universitat Autònoma de Barcelona (UAB), Passeig Maritim 25, 08003 Barcelona, Spain. ${ }^{2}$ Department of Respiratory Medicine, Parc de Salut MAR, Institut Hospital del Mar d'Investigacions Mèdiques (IMIM), Universitat Pompeu Fabra (UPF), CIBERES (ISC III), Barcelona, Spain

Received: 5 June 2018 Accepted: 18 September 2018

Published online: 01 October 2018

References

1. Laghi F, Tobin MJ. Disorders of the respiratory muscles. Am J Respir Crit Care Med. 2003;168:10-48.

2. Warner DO. Preventing postoperative pulmonary complications: the role of the anesthesiologist. Anesthesiology. 2000;92:1467-72.

3. Colucci DB, Fiore JF Jr, Paisani DM, Risso TT, Colucci M, Chiavegato LD, Faresin SM. Cough impairment and risk of postoperative pulmonary complications after open upper abdominal surgery. Respir Care. 2015;60: 673-8.

4. Man WD, Kyroussis D, Fleming TA, Chetta A, Harraf F, Mustfa N, Rafferty GF, Polkey MI, Moxham J. Cough gastric pressure and maximum expiratory mouth pressure in humans. Am J Respir Crit Care Med. 2003;168:714-7.

5. American Thoracic Society/European Respiratory S. ATS/ERS statement on respiratory muscle testing. Am J Respir Crit Care Med. 2002;166:518-624.

6. Gallart L, Gea J, Aguar MC, Broquetas JM, Puig MM. Effects of interpleural bupivacaine on respiratory muscle strength and pulmonary function. Anesthesiology. 1995;83:48-55.

7. Smith JA, Aliverti A, Quaranta M, McGuinness K, Kelsall A, Earis J, Calverley PM. Chest wall dynamics during voluntary and induced cough in healthy volunteers. J Physiol. 2012;590:563-74.

8. Chieveley-Williams S, Dinner L, Puddicombe A, Field D, Lovell AT, Goldstone JC. Central venous and bladder pressure reflect transdiaphragmatic pressure during pressure support ventilation. Chest. 2002;121:533-8. 
9. Collee GG, Lomax DM, Ferguson C, Hanson GC. Bedside measurement of intra-abdominal pressure (IAP) via an indwelling naso-gastric tube: clinical validation of the technique. Intensive Care Med. 1993;19:478-80.

10. Shafik A, El-Sharkawy A, Sharaf WM. Direct measurement of intra-abdominal pressure in various conditions. Eur J Surg. 1997;163:883-7.

11. Roca J, Sanchis J, Agusti-Vidal A, Segarra F, Navajas D, Rodriguez-Roisin R, Casan P, Sans S. Spirometric reference values from a Mediterranean population. Bull Eur Physiopathol Respir. 1986;22:217-24.

12. McGee DC, Gould MK. Preventing complications of central venous catheterization. N Engl J Med. 2003;348:1123-33.

13. Pittman JA, Ping JS, Mark JB. Arterial and central venous pressure monitoring Int Anesthesiol Clin. 2004;42:13-30.

14. Malbrain ML. Different techniques to measure intra-abdominal pressure (IAP): time for a critical re-appraisal. Intensive Care Med. 2004;30:357-71.

15. Iberti TJ, Lieber CE, Benjamin E. Determination of intra-abdominal pressure using a transurethral bladder catheter: clinical validation of the technique. Anesthesiology. 1989;70:47-50.

16. Cicchetti DV. Guidelines, criteria, and rules of thumb for evaluating normed and standardized assessment instruments in psychology. Psychol Assess. 1994;6:284-90

17. Bland JM, Altman DG. Statistical methods for assessing agreement between two methods of clinical measurement. Lancet. 1986;327(8476):307-10.

18. Enright PL, Beck KC, Sherrill DL. Repeatability of spirometry in 18,000 adult patients. Am J Respir Crit Care Med. 2004;169:235-8.

19. Lung function testing: selection of reference values and interpretative strategies. American Thoracic Society. Am Rev Respir Dis 1991;144:1202-18. https://doi.org/10.1164/ajrccm/144.5.1202.

20. Lee KK, Ward K, Rafferty GF, Moxham J, Birring SS. The intensity of voluntary, induced, and spontaneous cough. Chest. 2015;148:1259-67.

21. Byrd RB, Burns JR. Cough dynamics in the post-thoracotomy state. Chest. 1975;67:654-7.

22. Arora NS, Gal TJ. Cough dynamics during progressive expiratory muscle weakness in healthy curarized subjects. J Appl Physiol Respir Environ Exerc Physiol. 1981;51:494-8.

23. Rochester DF. Tests of respiratory muscle function. Clin Chest Med. 1988;9: 249-61.

24. Man WD, Moxham J, Polkey MI. Magnetic stimulation for the measurement of respiratory and skeletal muscle function. Eur Respir J. 2004;24:846-60.

25. Canet J, Gallart L, Gomar C, Paluzie G, Valles J, Castillo J, Sabate S, Mazo V, Briones Z, Sanchis J. Prediction of postoperative pulmonary complications in a population-based surgical cohort. Anesthesiology. 2010;113:1338-50.

Ready to submit your research? Choose BMC and benefit from:

- fast, convenient online submission

- thorough peer review by experienced researchers in your field

- rapid publication on acceptance

- support for research data, including large and complex data types

- gold Open Access which fosters wider collaboration and increased citations

- maximum visibility for your research: over $100 \mathrm{M}$ website views per year

At $\mathrm{BMC}$, research is always in progress.

Learn more biomedcentral.com/submissions 